\title{
Simple deformed Witt algebras ${ }^{1}$
}

\author{
Guang'ai Song*, Chunguang Xia ${ }^{\dagger}$ \\ * College of Mathematics and Information Science, Shandong Institute of Business \\ and Technology, Yantai, Shandong 264005, China \\ $\dagger$ Department of Mathematics, University of Science and Technology of China \\ Hefei 230026, China \\ E-mail: gasong@ccec.edu.cn, chgxia@mail.ustc.edu.cn
}

\begin{abstract}
For any factorization domain $\mathcal{A}$ and an algebra endomorphism $\sigma$ of $\mathcal{A}$, there exists a non-associative algebra $(\mathcal{A}, \sigma,[\cdot, \cdot])$ with multiplication satisfying skew-symmetry and generalized (twisted) Jacobi identities, called a $\sigma$-deformed Witt algebra. In this paper, we obtain the necessary and sufficient conditions for the algebra $(\mathcal{A}, \sigma,[\cdot, \cdot])$ to be simple.
\end{abstract}

Key words: Lie algebras; deformation; $\sigma$-derivations; Witt algebras 2000 MR Subject Classification: 17B66, 17B37

\section{$\S 1$. Introduction}

Deformation theory has many applications in mathematics and physics, especially in quantum theory (e.g., [D, ES, Kas]), therefore more and more attentions have been received on this field. For instance, the $q$-Witt algebras, $q$-Virasoro algebra, $q$-deformed Heisenberg algebras were investigated in $[\mathrm{H}, \mathrm{HS}]$, and the quantized structures of some Witt type algebras were present in $[\mathrm{G}, \mathrm{HW}$. Furthermore, there are various interesting deformations of Lie algebras, such as hom-Lie algebras, quasi-Lie algebras, quasi-hom-Lie algebras (e.g., [LS1, LS2, LS3, MS, RS, Y]). As a by-product, some algebras such as hom-Lie color algebras, hom-admissible algebras, hom-coalgebras, hom-Hopf algebras, etc. have also received some attentions in literature (e.g., [HLS, HS, LS1, LS2, LS3, MS, Y]).

The Witt algebra, which is the infinite-dimensional Lie algebra of linear differential operators on the circle, is as stated in [HLS] an important example in the classical differential and integral calculus, relating it to topology and geometry, and at the same time responsible for many of its key algebraic properties. It is also a well-known fact that the Virasoro algebra, which has played more and more important role in various mathematical and physical theories, such as vertex operator algebras, conformal field theory, the theory of the quantum Hall effect and integrable system, etc, is simply the universal central extension of the Witt algebra. Furthermore, the Witt type Lie coalgebras are the first examples of infinite dimensional Lie coalgebras constructed by Michaelis, and called "the gift given by God" [M]. Due

\footnotetext{
${ }^{1}$ Supported by NNSF grant 10871125, 11071147 of China, NSF grant Y2006A17, Y2008A04 of Shandong Province, NSF J06P52 of Shandong Provincial Education Department, China
} 
to the increasingly importance of Witt type algebras, generalizations of Witt type algebras and their subalgebras have been introduced and studied by many authors (e.g., [DZ1, DZ2, Kaw, O, P, S, SX1, SX2, SX3, SXZ, SZ, SZh, X]). In particular, the necessary and sufficient conditions for a wide class of Witt type algebras to be simple were obtained in $[\mathrm{P}]$, which can be conveniently used to verify whether or not a Witt type algebra is simple. From this, a large class of simple Witt type algebras were explicitly constructed in $[\mathrm{X}]$, whose structure and representation theories were studied in [SXZ, SZ, SZh].

Using $\sigma$-derivations, the authors in [HLS] obtained general deformations of Witt type algebras, referred to as $\sigma$-deformed Witt algebras. In order to construct explicitly simple $\sigma$ deformed Witt algebras and to study their structure and representation theories, it is desirable to establish some kind of criteria similar to $[\mathrm{P}]$ for Witt type algebras, to determine when a $\sigma$-deformed Witt algebra is simple. This is the aim of the present paper.

The main result of the present paper is the following theorem.

Theorem 1.1 Let $\mathcal{A}$ be a unique factorization domain over an arbitrary field $\mathbb{F}$, $\sigma$ an epimorphism on $\mathcal{A}$, and $\partial$ the $\sigma$-derivation defined by $(2.2)$ satisfying $\partial(\mathcal{A})=\mathcal{A}$. Then the $\sigma$-deformed Witt algebra $(\mathcal{A},[\cdot, \cdot], \sigma)$ defined in Theorem 2.2 is simple if and only if $\mathcal{A}$ is d-simple (cf. Definition 3.1).

\section{$\S 2$. Preliminary results}

Let $\mathbb{F}$ be an arbitrary field, $\mathcal{A}$ a unique factorization domain over $\mathbb{F}$, and $\sigma$ an endomorphism on $\mathcal{A}$. we first recall the notion of $\sigma$-derivation of $\mathcal{A}$ (see [Kas]), A linear map $d: \mathcal{A} \rightarrow \mathcal{A}$ is called a $\sigma$-derivation of $\mathcal{A}$ if

$$
d(a b)=d(a) b+\sigma(a) d(b) \text { for all } a, b \in \mathcal{A} .
$$

Denote the vector space of $\sigma$-derivations of $\mathcal{A}$ by $\mathcal{D}(\mathcal{A})$. It is easy to see that $d(1)=0$ for $d \in \mathcal{D}(\mathcal{A})$.

The following notions can be found in [HLS]. Assume that $\sigma \neq \operatorname{Id}$ (the identity map). Let $g=\operatorname{gcd}(\operatorname{Id}-\sigma)(\mathcal{A})$ be a greatest common divisor of $(\operatorname{Id}-\sigma)(\mathcal{A})$. Define a linear map $\partial: \mathcal{A} \rightarrow \mathcal{A}$ by

$$
\partial=\frac{\operatorname{Id}-\sigma}{g}, \text { i.e., } \partial(x)=\frac{x-\sigma(x)}{g} \text { for } x \in \mathcal{A} \text {. }
$$

The following result can be found in [HLS].

Lemma 2.1 The space $\mathcal{D}(\mathcal{A})$ is free of rank one as an $\mathcal{A}$-module with generator $\partial$.

Thus $\mathcal{D}(\mathcal{A})=\mathcal{A} \partial$, where an element $a \partial \in \mathcal{A} \partial$ acts on $\mathcal{A}$ as

$$
a \partial: x \rightarrow a \partial(x) \text { for } a, x \in \mathcal{A} \text {. }
$$


The algebra $(\mathcal{A}, \sigma,[\cdot, \cdot])$ with product defined below, is called a $\sigma$-deformed Witt algebra:

$$
[a, b]=\sigma(a) \partial(b)-\sigma(b) \partial(a) \text { for } a, b \in \mathcal{A}
$$

The following is one of the main results in [HLS].

Theorem 2.2 The $\sigma$-deformed Witt algebra $(\mathcal{A}, \sigma,[\cdot, \cdot])$ satisfies the following conditions:

(1) bilinearity: The operator $[\cdot, \cdot]: \mathcal{A} \times \mathcal{A} \rightarrow \mathcal{A}$ defined by (2.3) is bilinear.

(2) skew-symmetry: $[a, a]=0$ for all $a \in \mathcal{A}$.

(3) the generalized Jacobi identity:

$$
\begin{aligned}
0= & {[\sigma(a),[b, c]]+\delta[a,[b, c]] } \\
& +[\sigma(b),[c, a]]+\delta[b,[c, a]] \\
& +[\sigma(c),[a, b]]+\delta[c,[a, b]] \quad \text { for } a, b, c \in \mathcal{A},
\end{aligned}
$$

where, $\delta=\frac{\sigma(g)}{g}$.

Proof. The proof is straightforward.

Definition 2.3 A Hom-Lie algebra is a triple $(V,[\cdot, \cdot], \alpha)$ consisting of a vector space $V$, bilinear map $[\cdot, \cdot]: V \times V \rightarrow V$ and a linear map $\alpha: V \rightarrow V$ satisfying

$$
\begin{aligned}
& {[x, y]=-[y, x]} \\
& {[\alpha(x),[y, z]]+[\alpha(y),[z, x]]+[\alpha(z),[x, y]]=0,}
\end{aligned}
$$

for all $x, y, z \in V$.

Remark 2.4 (1) Since $g \mid(\mathrm{Id}-\sigma)(g)$ from the definition of $g$, we have $g \mid \sigma(g)$, and so $\delta=$ $\frac{\sigma(g)}{g} \in \mathcal{A}$. Thus the generalized Jacobi identity is well defined in $\mathcal{A}$. Furthermore, $\delta$ satisfies:

$$
\partial(\sigma(a))=\frac{(\operatorname{Id}-\sigma)(\sigma(a))}{g}=\frac{\sigma(a)-\sigma^{2}(a)}{g}=\frac{\sigma(g)}{g} \sigma\left(\frac{a-\sigma(a)}{g}\right)=\delta \sigma(\partial(a)),
$$

for $a \in \mathcal{A}$.

(2) If $\sigma \rightarrow 1$ (in some sense), the $\sigma$-derivation $\partial$ defined by (2.2) is the usual derivation on $\mathcal{A}$, and the bracket defined by (2.3) is the bracket of usual Witt type Lie algebras.

(3) If $\delta \in \mathbb{F}$, then by defining a linear map $\sigma_{1}: \mathcal{A} \rightarrow \mathcal{A}$ by $\sigma_{1}(a)=\sigma(a)+\delta a$ for $a \in \mathcal{A}$, and the bracket on $\mathcal{A}$ by $[a, b]=\sigma(a) \partial(b)-\sigma(b) \partial(a)$ for $a, b \in \mathcal{A}$, we obtain a Hom-Lie algebra $\left(\mathcal{A},[\cdot, \cdot], \sigma_{1}\right)$, called a Witt-type Hom-Lie algebra. 
(4) If $\delta \notin \mathbb{F}$, the triple $\left(\mathcal{A},[\cdot, \cdot], \sigma_{1}\right)$ is not a Hom-Lie algebra, in this case, the the triple $\left(\mathcal{A},[\cdot, \cdot], \sigma_{1}\right)$ is no longer satisfying Hom-Jacobi identity defined by Definition 2.3, and one can compute it directly.

\section{§3. The Proof of Main Result}

From now on, we shall discuss the conditions for $\sigma$-deformed Witt algebra $(\mathcal{A},[\cdot, \cdot], \sigma)$ to be simple. We shall always assume $\mathcal{A}$ is a unique factorization domain. As in the Lie algebra case (e.g., $[\mathrm{P}])$, we first give some definitions.

Definition 3.1 (1) An ideal $I$ of $\mathcal{A}$ is $(\sigma, \mathcal{D}(\mathcal{A})$ )-stable if $\sigma(a), d(a) \in I$ for all $a \in I, d \in$ $\mathcal{D}(\mathcal{A})$.

(2) The algebra $\mathcal{A}$ is $(\sigma, \mathcal{D}(\mathcal{A}))$-simple if it has no nontrivial $(\sigma, \mathcal{D}(\mathcal{A}))$-stable ideals.

Remark 3.2 (1) It is easy to see that if $I$ is a $(\sigma, \mathcal{D}(\mathcal{A}))$-stable ideal of $\mathcal{A}$, then $I$ is also an ideal of the $\sigma$-deformed Witt algebra $(\mathcal{A},[\cdot, \cdot], \sigma)$.

(2) By Lemma 2.1, $\mathcal{D}(\mathcal{A})=\mathcal{A} \partial$, thus an ideal $I$ of $\mathcal{A}$ is $\mathcal{D}(\mathcal{A})$-stable if and only if it is $\partial$-stable.

Similar to the usual Witt-type Lie algebra case (see $[\mathrm{P}]$ ), we have

Proposition 3.3 If $\mathcal{A}$ is $(\sigma, \mathcal{D}(\mathcal{A}))$-simple, then $\mathcal{A}^{\partial}=\{a \mid \partial(a)=0, a \in \mathcal{A}\}$ is $(\sigma, \partial)$-stable, moreover, $\mathcal{A}^{\partial}$ is a field containing $\mathbb{F}$.

Proof. From the definition of $\partial$, we see that $\partial(x)=0$ if and only if $\sigma(x)=x$. Thus $\mathcal{A}^{\partial}$ is obviously $\sigma$-stable. It is easy to see that $\mathcal{A}^{\partial}$ is a subring of $\mathcal{A}$. If $0 \neq a \in \mathcal{A}^{\partial}$, then $a \mathcal{A}$ is a nonzero $(\sigma, \mathcal{D}(\mathcal{A})$ )-stable ideal of $\mathcal{A}$, and so $a \mathcal{A}=\mathcal{A}$. Thus $a$ is an invertible element in $\mathcal{A}$. From $0=\partial(1)=\partial\left(a a^{-1}\right)=\partial(a) a^{-1}+\sigma(a) \partial\left(a^{-1}\right)=a \partial\left(a^{-1}\right)$, and since $\mathcal{A}$ has no nonzero divisors, we have $\partial\left(a^{-1}\right)=0$. Hence $a^{-1} \in \mathcal{A}^{\partial}$, namely, $\mathcal{A}^{\partial}$ is a field.

Lemma 3.4 If an ideal I of $\mathcal{A}$ is $\partial$-stable, then I is also $\sigma$-stable.

Proof. For any $x \in I$, since $\frac{x-\sigma(x)}{g}=\partial(x) \in I$, we obtain $\sigma(x)=x-g \partial(x) \in I$.

From Lemma 3.4 and Remark 3.2, we have,

$$
\begin{aligned}
\text { an ideal } I \text { of } \mathcal{A} \text { is }(\sigma, \mathcal{D}(\mathcal{A})) \text {-stable } & \Longleftrightarrow I \text { is } \partial \text {-stable, } \\
\qquad \mathcal{A} \text { is }(\sigma, \mathcal{D}(\mathcal{A})) \text {-simple } & \Longleftrightarrow \mathcal{A} \text { is } \partial \text {-simple. }
\end{aligned}
$$

Lemma 3.5 If $\sigma \neq 0$ and $\mathcal{A}$ is $\partial$-simple, then $\sigma$ is a monomorphism. 
Proof. Suppose that $\sigma$ is not a monomorphism, then $\operatorname{Ker}(\sigma) \neq\{0\}$ is an ideal of $\mathcal{A}$, which is obviously $\sigma$-stable. For any $x \in \operatorname{Ker}(\sigma)$, we have $\partial(x)=\frac{(\operatorname{Id}-\sigma)(x)}{g}=\frac{x}{g}$, and $\sigma(\partial(x))=\sigma\left(\frac{x}{g}\right)=$ $\frac{\sigma(x)}{\sigma(g)}=0$, and so $\partial(x) \in \operatorname{Ker}(\sigma)$. Thus $\operatorname{Ker}(\sigma)$ is a nontrivial $(\sigma, \partial)$-stable ideal of $\mathcal{A}$ by $(3.1)$, a contradiction.

Lemma 3.6 Let $V$ be a $\partial$-stable vector subspace of $\mathcal{A}$, and $\sigma$ an epimorphism of $\mathcal{A}$. Then any maximal ideal $I$ of $\mathcal{A}$ which is contained in $V$ is $(\sigma, \mathcal{D}(\mathcal{A}))$-stable.

Proof. It is easy to see that $I+\partial(I) \subset V$ is $\partial$-stable. For $x \in I, a \in \mathcal{A}$, from $\partial(a x)=$ $\partial(a) x+\sigma(a) \partial(x)$, we have $\sigma(a) \partial(x)=\partial(a x)-\partial(a) x \in I+\partial(I)$. Since $\sigma$ is an epimorphism of $\mathcal{A}$, we see that $I+\partial(I)$ is an ideal of $\mathcal{A}$. So $I+\partial(I) \subset I$ by the maximal nature of $I$. By $(3.1), I$ is $(\sigma, \mathcal{D}(\mathcal{A}))$-stable.

Lemma 3.7 Suppose $\mathcal{A}$ is $\partial$-simple. Let I be an ideal of $(\mathcal{A},[\cdot, \cdot], \sigma)$, and $\sigma$ an epimorphism on $\mathcal{A}$. Then $\partial(\mathcal{A}) \subset I$.

Proof. For $x \in I$, we have $\partial(x)=\sigma(1) \partial(x)-\sigma(x) \partial(1)=[1, x] \in I$, i.e., $I$ is a $\partial$-stable subspace of $\mathcal{A}$. For $x \in I, a \in \mathcal{A}$, we have $\sigma(x) \partial(a)-\sigma(a) \partial(x)=[x, a] \in I$. From this, we obtain

$$
\partial(x a)=\partial(x) a+\sigma(x) \partial(a) \equiv \partial(x) a+\sigma(a) \partial(x)(\bmod I) .
$$

Thus $\partial(x a) \in I \mathcal{A}$, i.e., $I \mathcal{A}$ is a $\partial$-stable ideal of $\mathcal{A}$.

Let $x$ be an element of $I$ with the shortest expression: $x=a_{x} x_{1} x_{2} \cdots x_{s}$, where $a_{x}$ is a unit of $\mathcal{A}$, and $x_{i}, i=1,2, \cdots, s$ are irreducible elements of $\mathcal{A}$ with $s$ being minimal. Then $x \mathcal{A}$ must be a maximal ideal of $\mathcal{A}$ which is contained in $I \mathcal{A}$. Thus $x \mathcal{A}$ is a $\partial$-stable ideal of $\mathcal{A}$ by Lemma 3.6. This implies that $x \mathcal{A}=\mathcal{A}$ since $\mathcal{A}$ is $\partial$-simple. Therefore $x$ is a unit of $\mathcal{A}$. For any $a \in \mathcal{A}$, we have

$$
\begin{aligned}
\sigma(x) x \partial(a) & =\sigma(x)(\partial(a) x+\sigma(a) \partial(x))-\sigma(a) \sigma(x) \partial(x) \\
& =\sigma(x) \partial(a x)-\sigma(a x) \partial(x) \\
& =[x, a x] \in I .
\end{aligned}
$$

This implies $\partial(a) \in I$ since $\sigma(x) x$ is also a unit. This proves $\partial(\mathcal{A}) \subset I$.

Now we can prove the main result of the paper.

Proof of Theorem 1.1. On one hand, if $\mathcal{A}$ is not $\partial$-simple, and $I$ is a $\partial$-stable proper ideal of $\mathcal{A}$, then one can easily check that $(I,[\cdot, \cdot], \sigma)$ is a proper ideal of $(\mathcal{A},[\cdot, \cdot], \sigma)$.

On the other hand, if $(I,[\cdot, \cdot], \sigma)$ is an ideal of $(\mathcal{A},[\cdot, \cdot], \sigma)$, then $\mathcal{A}=\partial(\mathcal{A}) \subset I$ by Lemma 3.7.

From Theorems 1.1 and 2.2, we obtain 
Theorem 3.8 Let $\mathcal{A}$ be a unique factorization domain over $\mathbb{F}, \sigma$ an epimorphism on $\mathcal{A}$, and $\partial$ the $\sigma$-derivation defined by $(2.2)$ satisfying $\partial(\mathcal{A})=\mathcal{A}$. Suppose $\delta$ defined in Theorem 2.2 is an element in $\mathbb{F}$. Then the Hom-Lie algebra $\left(\mathcal{A},[\cdot, \cdot], \sigma_{1}\right)$ with $\sigma_{1}$ being defined in Remark $2.4(3)$ is simple if and only if $\mathcal{A}$ is $\partial$-simple.

\section{§4. Applications}

As applications of Theorems 1.1 and 3.8, we obtain the following.

Corollary 4.1 Let $\mathcal{A}=\mathbb{C}[t]$ be the polynomial algebra in one variable $t$, and $\sigma$ the endomorphism of $\mathcal{A}$ determined by $\sigma(t)=q t$, where $0 \neq q \in \mathbb{C}$ is not a root of unit. Thus

$$
\sigma(f(t))=f(q t) \text { for } f(t) \in \mathcal{A}
$$

Define the $\sigma$-derivation by

$$
\partial(f(t))=\frac{(\mathrm{Id}-\sigma)(f(t))}{t-q t}=\frac{f(t)-f(q t)}{t-q t} .
$$

Then the $\sigma$-deformed Witt algebra $(\mathcal{A},[\cdot, \cdot], \sigma)$ is simple. Furthermore, the Hom-Lie algebra $\left(\mathcal{A},[\cdot, \cdot], \sigma_{1}\right)$ with $\sigma_{1}$ being defined in Remark $2.4(3)$ is simple.

Proof. It easy to check that $g=\operatorname{gcd}(\operatorname{Id}-\sigma)(\mathcal{A})=t-q t$, and $\partial(\mathcal{A})=\mathcal{A}$. Let $I \neq\{0\}$ be a $\partial$-stable ideal of $\mathcal{A}$. Since $\mathcal{A}$ is a principal ideal domain, we have $I=(p(t))$ for some $0 \neq p(t) \in \mathcal{A}$. Note that $\partial(p(t)) \in I=(p(t))$, i.e., $p(t) \mid \partial(p(t))$. On the other hand, we have $\operatorname{deg} \partial(p(t))<\operatorname{deg} p(t)$ by (4.1). This implies $\partial(p(t))=0$, namely, $p(t) \in \mathbb{C}$ is invertible. Thus $I=\mathcal{A}$, and $\mathcal{A}$ is $\partial$-simple. By Theorem $1.1,(\mathcal{A},[\cdot, \cdot], \sigma)$ is simple.

Since $\delta=\frac{\sigma(g)}{g}=\frac{\sigma(t)-\sigma(q t)}{t-q t}=q \in \mathbb{F}$, by Theorem 3.8, $\left(\mathcal{A},[\cdot, \cdot], \sigma_{1}\right)$ is a simple Hom-Lie algebra, where $\sigma_{1}$ is defined as in Remark 2.4(3) by $\sigma_{1}=\sigma+q \mathrm{Id}$.

Corollary 4.2 Let $\mathcal{A}, \sigma$, and $\partial$ be as in Corollary 4.1, and $q$ be an $n$-th primitive root of unit. Then the $\sigma$-deformed Witt algebra $(\mathcal{A},[\cdot, \cdot], \sigma)$ is not simple.

Proof. The principal ideal $I=\left(t^{n}\right)$ generated by $t^{n}$ is a proper $(\sigma, \partial)$-stable ideal of $\mathcal{A}$, and $(I,[\cdot, \cdot], \sigma)$ is a proper ideal of $(\mathcal{A},[\cdot, \cdot], \sigma)$. Thus $(\mathcal{A},[\cdot, \cdot], \sigma)$ is not simple.

Corollary 4.3 Let $\mathcal{A}=\mathbb{C}\left[t^{ \pm 1}\right]$ be the Laurent polynomial algebra in one variable $t$, and $\sigma$ the endomorphism on $\mathcal{A}$ determined by $\sigma(t)=q t$, where $0 \neq q \in \mathbb{C}$ is not a root of unit. Then the Hom-Lie algebra $\left(\mathcal{A},[\cdot, \cdot], \sigma_{1}\right)$ with $\sigma_{1}$ being defined in Remark $2.4(3)$ is simple. 
Proof. Since $(\operatorname{Id}-\sigma)(t)=t-q t=t(1-q)$ is a unit in $\mathcal{A}$, we see that $g=\operatorname{gcd}(\operatorname{Id}-\sigma)(\mathcal{A})$ is a unit of $\mathcal{A}$.

Take $g=t^{k}$ for any fixed $k \in \mathbb{Z}$. Then $\partial=t^{-k}(\operatorname{Id}-\sigma)$. It is easy to see that $\partial(\mathcal{A})=\mathcal{A}$. Let $\mathcal{I}$ be a nonzero $\partial$-stable ideal of $\mathcal{A}$ (thus also $\sigma$-stable). Let $0 \neq p(t)=\sum_{i=-m}^{n} a_{i} t^{i} \in \mathcal{I}$. By applying $\sigma$ to $p(t)$ several times, we obtain

$$
\sum_{i=-m}^{n} q^{i j}\left(a_{i} t^{i}\right)=\sigma^{j}(p(t)) \in \mathcal{I}, \quad j=0,1,2, \ldots, m+n .
$$

Regarding (4.2) as a system of linear equations on $(m+n+1)$ variables $a_{i} t^{i}, i=-m,-m+$ $1, \ldots, n$, since the determinant of coefficients is a nonzero Vandermonde determinant due to the fact that $q$ is not a root of unit, we obtain that each monomial (which is a unit in $\mathcal{A}$ ) of $p(t)$ is also in $\mathcal{I}$. Thus $\mathcal{I}=\mathcal{A}$. Let $\delta=\frac{\sigma(g)}{g}=q^{k}$, and $\sigma_{1}=\sigma+q^{k} \mathrm{Id}$, we obtain from Theorem 3.8 that $\left(\mathcal{A},[\cdot, \cdot], \sigma_{1}\right)$ is a simple Hom-Lie algebra.

Corollary 4.4 Let $\mathcal{A}=\mathbb{C}\left[t^{ \pm 1}\right]$ be the Laurent polynomial algebra in one variable $t$, and $\sigma$ the endomorphism of $\mathcal{A}$ defined by $\sigma(t)=q t^{s}$, where $0 \neq q \in \mathbb{C}, s \in \mathbb{Z}, s \neq 0,1,2$. Then the $\sigma$-deformed Witt algebra $(\mathcal{A},[\cdot, \cdot], \sigma)$ defined by Theorem 2.2 is not simple. Moreover, the triple $\left(\mathcal{A},[\cdot, \cdot], \sigma_{1}\right)$ is no longer a Hom-Lie algebra.

Proof. It is easy to see that $g$ is determined by the images of $t, t^{-1}$ on $\mathrm{Id}-\sigma$. Since

$$
(\operatorname{Id}-\sigma)(t)=t-q t^{s}=t\left(1-q t^{s-1}\right), \quad(\operatorname{Id}-\sigma)\left(t^{-1}\right)=t^{-1}-q^{-1} t^{-s}=-q^{-1} t^{-s}\left(1-q t^{s-1}\right)
$$

and $t,-q^{-1} t^{-s}$ are units of $\mathcal{A}$, we can take $g=1-q t^{s-1}$ if $s>2$, or $g=1-q^{-1} t^{1-s}$ if $s<0$.

If $s>2$, by denoting $T=q t^{s-1}$, we have

$$
\partial(T)=\frac{T-\sigma(T)}{1-T}=\frac{T-T^{s}}{1-T}=T\left(1+T+T^{2}+\cdots+T^{s-2}\right) .
$$

Hence the ideal $I$ generalized by $1+T+\cdots+T^{s-2}$ is a proper ideal of $\mathcal{A}$, which is easily checked to be $\partial$-stable (thus also $\sigma$-stable by Lemma 3.4). Therefore $I$ is a proper ideal of $\sigma$-deformed Witt algebra $(\mathcal{A},[\cdot, \cdot], \sigma)$, and so $(\mathcal{A},[\cdot, \cdot], \sigma)$ is not simple.

If $s<0$, by denoting $T=q^{-1} t^{1-s}$, we have

$$
\partial(T)=\frac{T-\sigma(T)}{1-T}=\frac{T-T^{s}}{1-T}=-T^{s} \frac{1-T^{1-s}}{1-T}=-T^{s}\left(1+T+\cdots+T^{-s}\right) .
$$

As above, the proper ideal $I$ of $\mathcal{A}$ generalized by $1+T+\cdots+T^{-s}$ is $(\sigma, \partial)$-stable, so $I$ is an ideal of $(\mathcal{A},[\cdot, \cdot], \sigma)$, and the algebra $(\mathcal{A},[\cdot, \cdot], \sigma)$ is not simple.

In both cases, since $\frac{\sigma(g)}{g} \notin \mathbb{F}$, the triple $\left(\mathcal{A},[\cdot, \cdot], \sigma_{1}\right)$ is not a Hom-Lie algebra by Remark $2.4(4)$. 
Corollary 4.5 Let $\mathcal{A}=\mathbb{C}\left[x_{1}^{ \pm 1}, x_{2}^{ \pm 1}, \cdots, x_{n}^{ \pm 1}\right]$ be the Laurent polynomial with $n$ variables, and $\sigma$ the endomorphism of $\mathcal{A}$ defined by $\sigma\left(x_{i}\right)=q_{i} x_{i}, i=1,2, \cdots, n$, where every $0 \neq q_{i} \in \mathbb{C}$ is not a root of unit. Then the triple $(\mathcal{A},[\cdot, \cdot], \sigma)$ is a simple $\sigma$-deformed Witt algebra, and the triple $\left(\mathcal{A},[\cdot, \cdot], \sigma_{1}\right)$ is a simple Hom-Lie algebra.

Proof. It easy to check that $\sigma$ is an epimorphism of $\mathcal{A}$, and we can take $g=\operatorname{gcd}(\operatorname{Id}-\sigma)(\mathcal{A})=1$. For any monomial $x_{1}^{k_{1}} x_{2}^{k_{2}} \cdots x_{n}^{k_{n}} \in \mathcal{A}$, since $\partial\left(x_{1}^{k_{1}} x_{2}^{k_{2}} \cdots x_{n}^{k_{n}}\right)=\left(1-q_{1}^{k_{1}} q_{2}^{k_{2}} \cdots q_{n}^{k_{n}}\right) x_{1}^{k_{1}} x_{2}^{k_{2}} \cdots x_{n}^{k_{n}}$, we see $\partial(\mathcal{A})=\mathcal{A}$. Let $I$ be any nonzero $\partial$-stable ideal of $\mathcal{A}$. As in (4.2), we can prove $I=\mathcal{A}$. Thus $\mathcal{A}$ is $\partial$-simple, and the triple $(\mathcal{A},[\cdot, \cdot], \sigma)$ is a simple $\sigma$-deformed Witt algebra. Since $\delta=1$, by Theorem 3.8, we obtain that the triple $\left(\mathcal{A},[\cdot, \cdot], \sigma_{1}\right)$ is a simple Hom-Lie algebra with $\sigma_{1}=\sigma+$ Id.

\section{References}

[D] V. Drinfel'd, Quantum groups, in "Proceeding of the International Congress of Mathematicians", Vol. 1, 2, Berkeley, Calif. 1986, Amer. Math. Soc., Providence, RI, 1987, pp. 789-820.

[DZ1] D.Ž. Doković, K. Zhao, Generalized Cartan type W Lie algebras in characteristic 0, J. Algebra 195 (1997), 170-210.

[DZ2] D.Ž. Doković, K. Zhao, isomorphisms, and second cohomology of generalized Witt algebras, Trans. Amer. Math. Soc. 350 (1998), 643-664.

[ES] P. Etingof, O. Schiffmann, Lectures on Quantum Groups, 2nd, International Press, USA, 2002.

[G] C. Grunspan, Quantizations of the Witt algebra and of simple Lie algebras in characteristic p, J. Algebra 280 (2004), 145-161.

[H] N. Hu, q-Witt Algebras, $q$-Virasoro Algebra, $q$-Lie Algebras, $q$-Holomorph Structure and Representations, Algebra Colloq. 6 (1999),No. 1, 51-70.

[HLS] J.T. Hartwig, D. Larsson, S.D. Silvestrov, Deformations of Lie algebras using $\sigma$-derivations, J. Algebra 295 (2006), no. 2, 314-361.

[HS] L. Hellström, S.D. Silvestrov, Commuting elements in q-deformed Heisenberg algebras, World Scientific Publishing Co., Inc., River Edge, NJ, 2000.

[HW] N. Hu, X. Wang, Quantizations of generalized-Witt algebra and of Jacobson-Witt algebra in modular case, J. Algebra 312 (2007), 902-929.

[Kas] C. Kassel, Quantum Groups, Graduate Texts in Mathematics, 155, Springer-Verlag, New York, 1995.

[Kaw] N. Kawamoto, Generalizations of Witt algebras over a field of characteristic zero, Hiroshima Math. J. 16 (1986), 417-462.

[LS1] D. Larsson, S.D. Silvestrov, Qusi-hom-Lie algebras, Central extensions and 2-cocycle-like identities, J. Algebra 288 (2005), 321-361.

[LS2] D. Larsson, S.D. Silvestrov, Quasi-Lie algebras, in "Noncommutative Geometry and Rep- 
resentation Theory in Mathematical Physics", Contemp, Math. 391 (2005), Amer. Math. Soc., Providence, RI, 241-248.

[LS3] D. Larsson, S.D. Silvestrov, Quasi-deformations of $s l_{2}(\mathbb{F})$ using twisting derivations, Comm. Algebra 35 (2007), 4303-4318.

[M] W. Michaelis, A Class of Infinite-dimensional Lie Bialgebras Containing the Virasoro Algebras, Adv. Math. 107 (1994), 365-392.

[MS] A. Makhlouf, S.D. Silvestrov, Hom-algebra structures, J. Gen. Lie Theory Appl. 2 (2008), 51-64.

[O] J.M. Osborn, New simple infinite-dimensional Lie algebras of characteristic 0, J. Algebra 185 (1996), 820-835.

[P] D.P. Passman, Simple Lie algebras of Witt Type, J. Algebra 206 (1998), 682-692.

[RS] L. Richard, S.D. Silvestrov, Qusi-Lie structure of $\sigma$-derivations of $\mathbb{C}\left[t^{ \pm 1}\right]$, J. Algebra 319 (2008), 1285-1304.

[Y] D. Yau, Enveloping algebras of hom-Lie algebras, J. Gen. Lie Theory Appl. 2 (2008), 95-108.

[S] Y. Su, Poisson brackets and structure of nongraded Hamiltonian Lie algebras related to locally-finite derivations, Canad. J. Math. 55 (2003), 856-896.

[SX1] Y. Su, X. Xu, Structure of divergence-free Lie algebras, J. Algebra 243 (2001), 557-595.

[SX2] Y. Su, X. Xu, Central simple Poisson algebras, Science in China A 47 (2004), 245-263.

[SX3] Y. Su, X. Xu, Structure of contact Lie algebras related to locally-finite derivations, Manuscripta Math. 112 (2003), 231-257.

[SXZ] Y. Su, X. Xu, H. Zhang, Derivation-simple Algebras and the Structures of Lie Algebras of Witt type, J. Algebra 233 (2000), 23-58.

[SZ] Y. Su, K. Zhao, Second cohomology group of generalized Witt type Lie algebras and certain representations, Comm. Algebra 30 (2002), 3285-3309.

[SZh] Y. Su, J. Zhou, Some representations of nongraded Lie algebras of generalized Witt type, $J$. Algebra 246 (2001), 721-738.

[X] X. Xu, New generalized simple Lie algebras of Cartan type over a field with characteristic 0, J. Algebra 224 (2000), 23-58. 